\title{
A Versão Breve da Escala de Empatia Básica numa Amostra Escolar de Jovens Portugueses: Validade, Fiabilidade e Invariância
}

\author{
The Short Version of the Basic Empathy Scale among a School Sample of Portuguese \\ Youths: Validity, Reliability and Invariance
}

\author{
Pedro Pechorro ${ }^{1}$, Saul Neves Jesus ${ }^{2}$, Rachel E. Kahn ${ }^{3}$, Rui Abrunhosa Gonçalves ${ }^{4}$ e Ricardo \\ Barroso 5
}

\begin{abstract}
Resumo
A empatia é um constructo multidimensional geralmente definido como a capacidade de entender e partilhar o estado emocional de outra pessoa. O objetivo do presente estudo consistiu em traduzir e validar a Escala de Empatia Básica versão breve adaptada (BES-A) numa amostra escolar $(N=543 ; M=15.65$ anos; $D P=1.84$ anos; amplitude $=12-20$ anos) de adolescentes portugueses do sexo masculino e feminino. A escala demonstrou adequadas propriedades psicométricas, nomeadamente em termos da sua estrutura bifatorial, consistência interna, validade discriminante com medidas de traços psicopáticos e de agressividade, além de associações com uma medida de ansiedade social. As associações com uma medida de ansiedade social revelaram correlações positivas significativas com a dimensão afetiva da empatia. As propriedades psicométricas encontradas justificam a utilização da BES-A com adolescentes portugueses.
\end{abstract}

Palavras-chave: avaliação, adolescencia, empatía, invariância de medida, validação

\begin{abstract}
Empathy is a multidimensional construct generally defined as the ability to understand and share another's emotional state. The main aim of the present study was to translate and validate the Basic Empathy Scale adapted short version (BES-A) among a school sample $(N=543 ; M=15.65$ years; $S D=1.84$ years; range $=12$ 20 years) of male and female Portuguese youths. The scale demonstrated good psychometric properties, namely in terms of its two-factor structure, internal consistency, discriminant validity with measures of psychopathic traits and aggression, and associations with a measure of social anxiety. Associations with a measure of social anxiety were also examined, revealing positive statistically significant correlations with the affective dimension of empathy. The psychometric properties of the BES-A justify its use among Portuguese adolescents.
\end{abstract}

Keywords: assessment, adolescence, empathy, measurement invariance, validation

A presente investigação foi parcialmente financiada pela Fundação para a Ciência e a Tecnologia (FCT) (Bolsa SFRH/BPD/86666/2012).

\footnotetext{
${ }^{1}$ Escola de Psicologia, Universidade do Minho. Campus de Gualtar, 4710-057 Braga, Portugal. Tel.: 253604267. Email: ppechorro@gmail.com

${ }^{2}$ Centro de Investigação sobre o Espaço e as Organizações, Universidade do Algarve. Campus de Gambelas, Edifício 9 , 8005-139 Faro, Portugal. Tel.: 289244406. E-mail: snjesus@ualg.pt

${ }^{3}$ Sand Ridge Secure Treatment Center. Research Unit, MMHI Bld 14, 301 Troy Drive, Madison, WI, 53704. USA. Tel.: 6083011359. E-mail: rachel.kahn@wisconsin.gov

${ }^{4}$ Escola de Psicologia, Universidade do Minho. Campus de Gualtar, 4710-057 Braga, Portugal. Tel.: 253604267. Email: rabrunhosa@psi.uminho.pt

${ }^{5}$ Departamento de Educação e Psicologia, Universidade de Trás-os-Montes e Alto Douro. Quinta de Prados, 5000-801

Vila Real. E-mail: rbarroso@utad.pt
}

Revista Iberoamericana de Diagnóstico y Evaluación - e Avaliação Psicológica. RIDEP · №49 · Vol.4 · 157-169 2018

ISSN: 1135-3848 print /2183-6051online 


\section{Introdução}

A empatia é considerada um constructo atual do ponto de vista da investigação sobre o desenvolvimento e comportamento social, que influencia, por exemplo, a adequada interação interpessoal e a prevenção da agressividade entre pares (Crocetti et al., 2016; Jolliffe \& Farrington, 2011). O constructo de empatia pode ser definido de diversas formas. Por exemplo, Cohen e Strayer (1996) definiram empatia como a compreensão e partilha do estado emocional de outra pessoa. Alguns autores (e.g., Jolliffe \& Farrington, 2006) defendem que o constructo da empatia pode ser subsumido em duas dimensões básicas: empatia afetiva e empatia cognitiva. A empatia afetiva é geralmente definida como o ressoar ou a congruência com o estado emocional de outra pessoa (Blair, 2005; Hoffman, 1987; Singer \& Lamm, 2009). A empatia cognitiva é frequentemente definida como a capacidade de tomar a perspetiva de outra pessoa, tal como ser capaz de imaginar o que outra pessoa poderá estar a sentir (Davis, 1980, 1983; Decety, 2010; Decety \& Cowell, 2014). O objetivo do presente artigo consistiu em validar a versão curta da Escala de Empatia Básica (BES; Jolliffe \& Farrington, 2006) numa amostra escolar de adolescentes portugueses de forma a disponibilizar uma versão mais acessível e mais rápida de preencher que mantenha boas propriedades psicométricas.

As diferenças entre rapazes e raparigas relativamente à empatia têm sido descritas desde uma idade muito precoce. De acordo com McClure (2000), raparigas de 3 a 4 meses são capazes de discriminar expressões faciais melhor que rapazes no paradigma de still-face materno. Adicionalmente, ao longo da infância e adolescência, as raparigas tendem a demonstrar níveis mais altos de empatia e seus correspondentes pró sociais (Chaplin \& Aldao, 2013; Zacarías-Salinas, \& Andrade-Palos, 2014). A transição para a puberdade amplia ainda mais as diferenças entre rapazes e raparigas (Lam, Solmeyer, \& McHale, 2012), sugerindo que esta fase pode ser um período importante para o desenvolvimento da empatia. De facto, segundo alguns autores a empatia atinge o seu pico de desenvolvimento durante a fase final da adolescência (Hoffman, 1987). As diferenças entre os sexos parecem ser estáveis ao longo do ciclo de vida (Michalska, Kinzler, \& Decety, 2013), com o sexo feminino a demonstrar consistentemente níveis de empatia mais altos, evidenciando-se também que quem demonstra precocemente níveis de empatia altos tende a mantê-los ao longo da vida (Eisenberg et al., 1999).

Dada a importância do constructo de empatia, uma medida que consiga capturar as duas dimensões componentes da empatia pode ser bastante útil. Uma ampla variedade de medidas de autorresposta de empatia foram desenvolvidas ao longo dos anos, incluindo a Hogan Empathy Scale (HES; Hogan, 1969), o Interpersonal Reactivity Index (IRI; Davis, 1980), e a Balanced Empathy Emotional Scale (BEES; Mehrabian, 1996). Todavia, estas medidas de empatia revelam algumas limitações (e.g., confundem simpatia com empatia, não captam adequadamente a dimensão cognitiva da empatia, foram validadas apenas em estudantes universitários).

Jolliffe e Farrington (2006) desenvolveram a Escala de Empatia Básica (BES) na tentativa de ultrapassar as limitações dos instrumentos concebidos previamente. Durante o processo de desenvolvimento da BES, estes autores administraram 40 itens relacionados com empatia a uma amostra mista de 363 adolescentes que frequentavam o liceu em Inglaterra. A análise fatorial exploratória (EFA) revelou uma solução de dois fatores composta por 20 itens que mediam empatia afetiva e cognitiva. Posteriormente esta estrutura foi replicada usando análise fatorial confirmatória (CFA). A consistência interna por alfa de Cronbach revelou-se boa para a dimensão afetiva $($ alfa $=.85)$ e para a dimensão cognitiva (alfa=.79). Foi também demonstrada a validade de constructo, obtendo-se as correlações esperadas com outras medidas de empatia e de personalidade (Jolliffe \& Farrington, 2006).

Desde então, a BES tem sido validada numa variedade de amostras escolares e comunitárias de países como França (D’Ambrosio, Olivier, Didon, \& Besche, 2009), Itália (Albiero, Matricardi, Speltri, \& Toso, 2009), Singapura (Ang \& Goh, 2010), Turquia (Topçu \& Erdur-Baker, 2012), China (Geng, Xia, \& Qin, 2012), e Espanha (Sánchez-Pérez, Fuentes, Jollife, \& GonzálezSalinas, 2014; Villadangos, Errasti, Amigo, 
Jolliffe, \& Garcia-Cueto, 2016). Tais estudos têm vindo a suportar de forma consistente a estrutura de dois fatores, com a consistência interna a variar de marginal a boa. Adicionalmente, em amostras diferentes a BES tem demonstrado as esperadas associações positivas com outras escalas de empatia (Albiero et al., 2009; D'Ambrosio et al., 2009) e medidas de comportamentos pró sociais (Geng et al., 2012), além de associações negativas com medidas de perturbações internalizantes (D’Ambrosio et al., 2009) e problemas emocionais (Geng et al., 2012). Mais recentemente, a BES foi investigada em Portugal (Anastácio, Vagos, Nobre-Lima, Rijo, \& Jolliffe, 2016) usando uma amostra comunitária nãorepresentativa e não-diversificada geograficamente, sendo que esta versão ficou com 16 itens de forma a conseguir obter um modelo de dois fatores que fosse aceitável.

A BES também foi validada numa variedade de amostras forenses e de jovens em risco compostas por jovens do sexo masculino. SalasWright, Olate, e Vaughn (2012), utilizando uma amostra mista de jovens hispânicos em risco de El Salvador envolvidos em gangues, encontraram resultados que suportavam uma versão breve adaptada da BES com sete itens (BES-A). SalasWright et al. (2012) utilizaram um combinação de vários passos sequenciais de forma a chegarem à BES-A, nomeadamente remoção de itens com assimetrias e/ou curtoses acentuadas, com correlações item-total corrigidas baixas $(<.20)$ ou negativamente correlacionados com outros itens da escala (incluindo itens formulados na negativa). Seguidamente procederam a análise da fiabilidade através de Alfa de Cronbach e finalmente a análise fatorial confirmatória, incluindo invariância de medida. Desta forma a BES-A ficou composta por sete itens, dos quais três relativos a empatia afetiva e quatro relativos a empatia cognitiva.

Em Portugal, Pechorro, Ray, Salas-Wright, Maroco, e Gonçalves (2015), utilizando uma amostra de jovens delinquentes do sexo masculino internados em Centro Educativo, encontraram resultados em termos de validade e fiabilidade que apoiaram tanto a versão original de 20 itens como a versão breve de sete itens. Mais recentemente Pechorro, Kahn, Gonçalves e Ray (2017) utilizando uma amostra mista composta por raparigas internadas em Centro Educativo e raparigas provenientes de contexto escolar verificaram que a versão original da BES obtinha um bom ajustamento apenas na amostra escolar. De salientar que a tradução utilizada por Pechorro et al. $(2015$; 2017) é distinta da tradução de Anastácio et al. (2016), tendo ambas sido devidamente autorizadas pelo autor principal da BES (Jolliffe \& Farrington, 2006) mas efetuadas de forma independente uma da outra. Na Alemanha, Heynen, van der Helm, Stams e Korebrits (2016), usando uma amostra de jovens delinquentes detidos, encontraram suporte para uma versão de 12 itens da BES na qual estavam incluídos os sete itens da versão curta.

Os estudos que utilizaram a BES também têm contribuído para corroborar o fato de as mulheres tenderem a pontuar mais alto em empatia, com as dimensões de efeito a serem mais elevadas na dimensão de empatia afetiva (Albiero et al., 2009; Anastácio et al., 2016; D’Ambrosio et al., 2009; Geng et al., 2012; Jolliffe \& Farrington, 2006; Salas-Wright et al., 2012). Não foram encontradas diferenças entre os sexos na estrutura fatorial da $\mathrm{BES}$, apesar de ser notório que frequentemente certos itens têm de ser removidos devido a baixas saturações e baixas correlações item-total. Todavia, enquanto a investigação passada se tem focado na versão original da BES, tanto quanto é do nosso conhecimento ainda não foi examinada a versão adaptada breve de sete itens designada por BES-A em amostras escolares de rapazes e raparigas.

Desta forma, o principal objetivo deste estudo consistiu na validação da BES-A numa amostra escolar geograficamente diversificada de grande dimensão constituída por rapazes e raparigas portugueses, incluindo a testagem da invariância de medida. Colocámos as hipóteses de que: 1) a estrutura de dois fatores da BES-A seria confirmada e haveria invariância de medida entre rapazes e raparigas; 2) seria demostrada boa consistência interna medida por alfa de Cronbach e coeficiente Omega; 3) a BES-A demonstraria validade discriminante com medidas de traços psicopáticos e de agressão, e associações positivas com uma medida de ansiedade social; e de que 4) as raparigas obteriam pontuações mais elevadas na BES-A que os rapazes. 


\section{Método}

\section{Participantes}

A amostra composta por 543 participantes $(M=15.65$ anos; $D P=1.84$ anos; amplitude=12-20 anos), provenientes de estabelecimentos públicos de ensino da grande Lisboa, Algarve e de Coimbra, foi subdividida em rapazes $(n=269$; $M=15.80$ anos; $D P=1.81$ anos; amplitude $=12-20$ anos) e raparigas $(n=274 ; \quad M=15.50$ anos; $D P=1.86$ anos; amplitude $=12-20$ anos). Não foram encontradas diferenças estatisticamente significativas entre rapazes e raparigas relativamente a idade $(F=3.52 ; p=.06)$, anos de escolaridade completados $(F=5.53, \quad p=.12 ; M$ rapazes $=9.01, \quad D P \quad$ rapazes $=1.47 ; \quad M$ raparigas $=8.87, D P$ raparigas $=1.48$ ) e etnia $\left(\chi^{2}=6.69, p=.12\right)$.

\section{Medidas}

A Escala de Empatia Básica (Basic Empathy Scale - BES; Jolliffe \& Farrington, 2006) é uma medida de autorresposta constituída por 20 itens, desenhada para medir duas dimensões da empatia em adolescentes: empatia afetiva (11 itens) e empatia cognitiva (9 itens). Cada item é cotado em escala ordinal de 5 pontos, de Discordo totalmente a Concordo totalmente. Pontuações mais elevadas indicam níveis de empatia mais elevados. Na presente investigação foi utilizada a versão portuguesa da BES e a sua correspondente versão breve de sete itens designada por BES-A que mantém a estrutura de dois fatores (Pechorro, Ray et al, 2015; Pechorro, Kahn et al., 2017). Os itens constituintes da BES-A são disponibilizados na secção de Resultados do presente artigo.

O Dispositivo de Despiste de Processo Antissocial (Antisocial Process Screening Device - APSD; Frick \& Hare, 2001) versão de autorresposta (APSD-SR; Caputo, Frick, \& Brodsky, 1999) é um instrumento constituído por 20 itens modelado a partir da Psychopathy Checklist (Hare, 2003; Forth, Kosson, \& Hare, 2003; Pechorro, Barroso, Maroco, Vieira, \& Gonçalves, 2015) para medir traços psicopáticos em adolescentes. Cada item é cotado em escala ordinal de 3 pontos, de Nunca a Frequentemente. O APSD-SR tem três fatores: Impulsividade, Narcisismo e Calosidade/Insensibilidade emocional. Pontuações mais elevadas indicam níveis de traços psicopáticos mais elevados. $\mathrm{Na}$ presente investigação foi utilizada a versão portuguesa do APSD-SR (Pechorro, Hidalgo, Nunes, \& Jiménez, 2016; Pechorro, Gonçalves, Andershed, \& DeLisi, no prelo) que revelou propriedades psicométricas em termos de validade e fiabilidade semelhantes às do APSD original que podem ser consideradas adequadas. $\mathrm{O}$ coeficiente alfa do APSD-SR na presente investigação foi .77 .

O Inventário de Traços Psicopáticos para Jovens (Youth Psychopathic Traits Inventory short version - YPI-S; van Baardewijk et al., 2010) é uma versão breve do YPI original (Andershed, Kerr, Stattin, \& Levander, 2002) concebido para medir traços psicopáticos em adolescentes. Os 18 itens que o constituem são cotados numa escala ordinal de 4 pontos, de Não se aplica a Aplica-se muito bem. O YPI-S tem uma estrutura de três fatores semelhante à do YPI original composta pelas dimensões comportamental, afetiva e interpessoal. Pontuações mais elevadas indicam maior presença de traços psicopáticos. Na presente investigação foi utilizada a versão portuguesa do YPI-S (Pechorro, Andershed, Ray, Maroco, \& Gonçalves, 2015; Pechorro, Ribeiro da Silva, Rijo, Gonçalves, \& Andershed, 2017) que revelou propriedades psicométricas em termos de validade e fiabilidade semelhantes às do YPI-S original que podem ser consideradas satisfatórias. $\mathrm{O}$ coeficiente alfa do YPI-S na presente investigação foi 92 .

O Inventário de Traços Calosos/Insensibilidade emocional (Inventory of Callous-Unemotional Traits - ICU; Essau et al., 2006; Kimonis et al., 2008) é um instrumento de autorresposta constituído por 24 itens que avalia traços calosos/frieza emocional. Cada item é cotado em escala ordinal de 4 pontos, de Totalmente falso a Totalmente verdade. O ICU tem uma estrutura de três fatores composta pelas dimensões: Calosidade/Frieza emocional, Insensibilidade emocional e Indiferença emocional. Pontuações mais elevadas indicam maior presença de traços calosos/frieza emocional. Na presente investigação foi utilizada a versão portuguesa do ICU (Pechorro, Ray, Gonçalves, \& Jesus, 2017; Pechorro, Gonçalves, Hawes, \& Ray, no prelo) que revelou 
propriedades psicométricas em termos de validade e fiabilidade semelhantes às do ICU original que podem ser consideradas satisfatórias. $\mathrm{O}$ coeficiente alfa do ICU na presente investigação foi .88

O Questionário de Agressividade ReativaProativa (Reactive-Proactive Aggression Questionnaire - RPQ; Raine et al., 2006) é uma medida de autorresposta constituída por 23 itens que distingue entre agressividade reativa $\mathrm{e}$ proativa. Cada item é cotado em escala ordinal de 3 pontos, de Nunca a Frequentemente. O RPQ pode ser utilizado com adolescentes e jovens adultos. Pontuações mais elevadas indicam maior presença dos traços em questão. Na presente investigação foi utilizada a versão portuguesa do RPQ (Pechorro, Ray, Raine, Maroco, \& Gonçalves, 2017; Pechorro, Kahn, Ray, Raine, \& Gonçalves, 2017) que revelou propriedades psicométricas em termos de validade e fiabilidade semelhantes às do RPQ original que podem ser consideradas satisfatórias. O coeficiente alfa do RPQ na presente investigação foi .86 .

A Escala de Ansiedade Social para Adolescentes (Social Anxiety Scale for Adolescents - SAS-A; La Greca \& Lopez, 1998) é uma medida de autorresposta constituída por 22 itens (dos quais 4 são itens neutros não cotados) que avalia as experiências de ansiedade social dos adolescentes no contexto das relações com os seus pares. Os itens são avaliados segundo uma escala ordinal de 5 pontos, que vai de De forma nenhuma a Todas as vezes. Para além da pontuação total pode-se obter também as pontuações nas três subescalas, nomeadamente: Medo de avaliação negativa, Evitação e mal-estar social - Novo e Evitação e mal-estar social - Geral (La Greca \& Lopez, 1998). Pontuações mais elevadas indicam níveis de ansiedade social mais elevados. $\mathrm{Na}$ presente investigação foi utilizada a versão portuguesa da SAS-A (Pechorro, Ayala-Nunes, Nunes, Maroco, \& Gonçalves, 2016) que revelou propriedades psicométricas em termos de validade e fiabilidade semelhantes às das SAS-A original que podem ser consideradas satisfatórias. $O$ coeficiente alfa da SAS-A na presente investigação foi .92 .

Adicionalmente, foi construído um questionário ad hoc para descrever as caraterísticas sociodemográficas dos participantes, que incluiu variáveis como idade, sexo, escolaridade e etnia.

\section{Procedimentos}

Solicitou-se e obteve-se autorização ao autor principal da BES (Jolliffe \& Farrington, 2006) para traduzir e utilizar o instrumento em Portugal. Durante o processo de tradução e adaptação da BES seguiram-se recomendações estabelecidas internacionalmente (Hambleton, Merenda, \& Spielberger, 2005). O primeiro autor e o penúltimo autor deste artigo efetuaram a tradução da escala. De seguida um tradutor bilingue fez a respetiva retroversão para inglês, que foi então comparada com o instrumento original. Foi então realizado um estudo pré-teste para analisar a qualidade da tradução, detetar problemas eventuais problemas e aperfeiçoar a linguagem de forma a torná-la mais facilmente entendível. Chegou-se assim à versão final da escala (para uma descrição mais detalhada ver Pechorro, Ray et al., 2015).

A recolha dos questionários decorreu em escolas básicas/secundárias da região da grande Lisboa, Algarve e de Coimbra após se ter obtido autorização por parte da Direção-Geral de Educação (DGE). Foi entregue um termo de consentimento assinado pelo encarregado de educação de cada aluno autorizando a participação na investigação. A aplicação decorreu em contexto de grupo. A taxa de participação foi de aproximadamente $85 \%$.Foram excluídos os participantes que estavam fora do intervalo etário estabelecido ou que entregaram questionários não preenchidos, incompletos ou ilegíveis.

O software SPSS v25 (IBM SPSS, 2017) e o software EQS 6.3 (Bentler \& Wu, 2015) foram utilizados para inserir e analisar os dados. No tratamento de dados estatísticos recorreu-se a estatísticas descritivas, ANOVA, qui-quadrado, análise fatorial confirmatória, análise de consistência interna (por alfa de Cronbach e Omega) e correlações paramétricas e nãoparamétricas.

A análise da estrutura fatorial da BES-A foi efetuada no software EQS. Os índices de ajustamento calculados incluíram: Qui-quadrado de Satorra-Bentler/graus de liberdade, CFI (Comparative Fit Index - Índice de ajustamento comparativo), IFI (Incremental Fit Index - Índice 
de ajustamento incremental), RMSEA (Root Mean Square Error of Approximation - Raiz quadrada do erro médio de aproximação). Um valor de quiquadrado/graus de liberdade $<5$ é considerado adequado, se $\leq 2$ é considerado bom e se $=1$ é considerado muito bom (Maroco, 2014; West, Taylor, \& Wu, 2012). Valores CFI $\geq .90$ e RMSEA $<.10$ indicam ajustamento adequado; valores de $C F I \geq .95$ e RMSEA $\leq .06$ indicam um ajustamento bom (Byrne, 2006). Um valor de IFI $\geq .90$ é considerado aceitável. Foram considerados índices de modificação para melhorar o ajustamento do modelo caso necessário. A CFA foi efetuada diretamente nos itens utilizando valores de cargas fatoriais $\geq .45$.

Foram testados vários modelos. $\mathrm{O}$ primeiro foi um modelo de um fator em que todos os itens saturam num único fator latente. $\mathrm{O}$ segundo foi um modelo de dois fatores inter-correlacionados em que os itens saturam nos respetivos fatores. $\mathrm{O}$ terceiro foi um modelo de dois fatores com um fator hierárquico superior de segunda ordem. Em todos os modelos testados o Coeficiente de Mardia situou-se acima de 5, indicando uma distribuição não normal, pelo que se optou pela utilização de matriz de correlações policóricas com métodos de estimação robustos nos itens ordinais dado que proporcionam melhores resultados (Byrne, 2006).

A invariância de medida foi examinada separando a amostra total em masculina $\mathrm{e}$ feminina. O habitual teste de diferença de $\mathrm{S}-\mathrm{B} \chi^{2}$ (Millsap \& Olivera-Aguilar, 2012), o $\triangle$ CFI e o $\triangle$ RMSEA (Chen, 2007; Cheung \& Rensvold, 2002) foram utilizados para determinar o ajustamento do modelo. Uma folha Excel fornecida por Bryant e Satorra (2012) foi utilizada para calcular o teste de diferença (http://www.econ.upf.edu/ satorra/).

Foram utilizadas ANOVAs e testes de quiquadrado para comparar os grupos de rapazes e raparigas quando as variáveis eram métricas e nominais, respetivamente. Foram utilizadas correlações Pearson para analisar as associações entre as variáveis métricas que apresentaram distribuições aproximadamente normais utilizando critérios de assimetria e de curtose (Leech, Barrett, \& Morgan, 2015; Maroco, 2014), incluindo as existentes entre a BES-A e as suas dimensões para examinar a validade de constructo. Em termos de magnitude de correlações, consideraram-se correlações fracas as correlações entre 0 e .20 , correlações moderadas entre .20 e .50 , e correlações fortes acima de .50 (Ferguson, 2009; Lipsey, 1998). As pontuações totais das escalas, de acordo com o que é tradicionalmente efetuado em psicometria (e.g., Nunnally \& Bernstein, 1994), foram utilizadas para examinar a validade discriminante e associações com a ansiedade social. A consistência interna por alfa de Cronbach e Omega foi considerada marginalmente aceitável se entre .60 e .69 , aceitável se entre .70 e .79 e boa se acima de .80 (DeVellis, 1991; Dunn, Baguley, \& Brunsden, 2014; Nunnally, \& Bernstein, 1994). As médias das correlações inter-item (MCII) foram consideradas adequadas se entre .15 e .50 , enquanto as correlações item-total corrigidas (ACITC) foram consideradas adequadas se acima de .30 (Clark \& Watson, 1995; Nunnally \& Bernstein, 1994).

\section{Resultados}

O primeiro passo para avaliar as propriedades psicométricas da versão portuguesa da BES-A consistiu em tentar replicar por meio de CFA os diferentes modelos possíveis para este instrumento. $\mathrm{O}$ modelo de um fator também foi analisado de forma a compará-lo com os restantes modelos. No Quadro 1 encontram-se os índices de ajustamento obtidos na amostra total, amostra masculina e amostra feminina. $\mathrm{O}$ modelo de dois fatores e o modelo de dois fatores de segunda ordem obtiveram apoio adequado em termos dos índices de ajustamento.

No Quadro 2 são reportadas as saturações do modelo de dois fatores de segunda ordem nas diferentes amostras. Conforme pode ser observado as saturações estiveram acima de .45 .

$\mathrm{O}$ passo seguinte consistiu em testar a invariância de medida em rapazes e raparigas. $\mathrm{O}$ modelo base foi testado em termos de invariância métrica (ou fraca) e invariância forte (ou escalar), apresentando-se os resultados no Quadro 3. Os valores de $\Delta \mathrm{S}-\mathrm{B} \chi 2(\mathrm{df})$ foram sempre significantes, mas se considerarmos os critérios de Chen (2007) e de Cheung e Rensvold (2002), nomeadamente, $\triangle$ CFI abaixo de .01, $\triangle$ RMSEA abaixo de .015 , há suporte para a existência de 
Quadro 1. Índices de ajustamento da BES-A

\begin{tabular}{|c|c|c|c|c|c|}
\hline & $\mathrm{S}-\mathrm{B} \chi^{2} / \mathrm{gl}$ & IFI & CFI & $\begin{array}{l}\text { RMSEA } \\
\text { (90\% C.I.) }\end{array}$ & AIC \\
\hline \multicolumn{6}{|l|}{ Amostra total } \\
\hline 1-fator & 19.35 & .90 & .90 & $.15(.14 .17)$ & 243.61 \\
\hline 2-fatores & 3.35 & .99 & .99 & $.06(.04-.07)$ & 17.49 \\
\hline 2 -fatores $2^{\mathrm{a}}$ ordem & 3.5 & .98 & .98 & $.06(.04-.08)$ & 16.50 \\
\hline \multicolumn{6}{|l|}{ Masculina } \\
\hline 1-fator & 24.79 & .76 & .76 & $.25(.23-.28)$ & 319.10 \\
\hline 2-fatores & 3.55 & .98 & .98 & $.08(.06-.11)$ & 20.15 \\
\hline 2 -fatores $2^{\mathrm{a}}$ ordem & 3.21 & .97 & .97 & $.08(.05-.11)$ & 13.27 \\
\hline \multicolumn{6}{|l|}{ Feminina } \\
\hline 1-fator & 14.93 & .85 & .85 & $.18(.16-.21)$ & 181.18 \\
\hline 2-fatores & 3.60 & .98 & .98 & $.08(.06-.11)$ & 20.87 \\
\hline 2 -fatores $2^{a}$ ordem & 3.54 & .97 & .97 & $.08(.05-.11)$ & 16.94 \\
\hline
\end{tabular}

Nota. $\mathrm{S}-\mathrm{B} \chi^{2} / \mathrm{gl}=$ qui-quadrado de Satorra-Bentler/graus de liberdade; IFI = Índice de ajustamento incremental; $\mathrm{C} F \mathrm{I}=$ Índice de ajustamento comparativo; RMSEA $(90 \% \mathrm{CI})=$ Raiz quadrada do erro médio de aproximação (intervalo de confiança a 90\%)

Quadro 2. Cargas fatoriais da BES-A

\begin{tabular}{lc}
\hline Itens & T/M/F \\
\hline BES-A Afetiva & $.79 / .69 / .57$ \\
1(2). Depois de falar com um amigo que está triste geralmente também fico triste. & $.55 / .88 / .84$ \\
2(5). Sou facilmente influenciado pelos sentimentos das outras pessoas. & $.53 / .69 / .78$ \\
3(17). Costumo deixar-me influenciar pelos sentimentos dos meus amigos. & $.76 / .73 / .80$ \\
BES-A Cognitiva & $.79 / .81 / .82$ \\
4(9). Quando as pessoas se sentem em baixo geralmente costumo perceber como elas se sentem. & $.83 / .83 / .84$ \\
5(10). Geralmente costumo perceber quando os meus amigos estão nervosos. & $.68 / .66 / .71$ \\
6(12). Geralmente costumo perceber como as pessoas se sentem mesmo antes de elas me dizerem. & \\
7(14). Geralmente costumo perceber quando as pessoas estão contentes. &
\end{tabular}

Nota. M/F=Amostras Total/Masculina/Feminina

Quadro 3. Invariância de medida da BES-A

\begin{tabular}{lcccc}
\hline Modelo & $\mathrm{S}-\mathrm{B} \chi^{2}(\mathrm{gl})$ & $\Delta \mathrm{S}-\mathrm{B} \chi^{2}(\mathrm{gl})$ & $\mathrm{CFI}$ & $\begin{array}{c}\text { RMSEA (90\% } \\
\text { C.I.) }\end{array}$ \\
\hline Modelo base (configural) & $76.25(26)$ & -- & .98 & $.07(.05-.09)$ \\
Invariância fraca (métrica) & $86.10(31)$ & $11.84(5)^{*}$ & .98 & $.07(.05-.08)$ \\
Invariância forte (escalar) & $89.92(34)$ & $15.34(8)^{*}$ & .98 & $.07(.05-.08)$ \\
\hline
\end{tabular}

Nota. $\mathrm{S}-\mathrm{B} \chi^{2} / \mathrm{gl}=$ qui-quadrado de Satorra-Bentler/graus de liberdade; $\mathrm{CFI}=$ Índice de ajustamento comparativo; RMSEA $(90 \% \mathrm{CI})=$ Raiz quadrada do erro médio de aproximação (intervalo de confiança a $90 \%$ )

$* p \leq .05$

invariância de medida fraca (métrica) e forte (escalar) entre rapazes e raparigas.

O Quadro 4 apresenta as correlações entre a BES-A e a BES original. Tal como esperado as correlações foram positivas e estatisticamente significativas.

No Quadro 5 são apresentados os valores de consistência interna na amostra masculina e feminina. Os valores obtidos podem ser considerados satisfatórios.

No Quadro 6 são apresentadas as correlações da BES-A com o APSD-SR, o YPI-S, o ICU e o RPQ em termos de validade discriminante. São também apresentadas as correlações com a SAS-A, que mede ansiedade social.

Em termos de validade de grupos conhecidos, a comparação de rapazes e raparigas revelou que as raparigas pontuam significativamente mais alto em termos do total da BES-A ( $F=51.657, p \leq .001$, $\eta_{p}{ }^{2}=.06, \quad$ potência $=1.0 ; \quad M$ rapazes=13.79, $D P$ rapazes $=4.83 ; \quad M \quad$ raparigas $=16.23, \quad D P$ raparigas $=4.67)$, da dimensão Afetiva $(F=42.160$, $p \leq .001, \eta_{p}{ }^{2}=.05$, potência $=1.0 ; M$ rapazes $=4.26$, $D P$ rapazes $=2.71) ; \quad M$ rapariga $=5.50, D P$ raparigas $=2.63$ ), e da dimensão Cognitiva $\left(F=26.185, \quad p \leq .001, \quad \eta_{p}{ }^{2}=.03\right.$, potência $=.99 ; \quad M$ rapazes $=9.53, \quad D P \quad$ rapazes $=3.29 ; \quad M$ raparigas $=10.73, D P$ raparigas $=3.26$ ). 
Quadro 4. Matriz de correlações de Pearson da BES-A e BES original

\begin{tabular}{|c|c|c|c|c|c|c|}
\hline & BES & $\begin{array}{c}\text { BES } \\
\text { Afetiva }\end{array}$ & $\begin{array}{c}\text { BES } \\
\text { Cognitiva }\end{array}$ & BES-A & $\begin{array}{l}\text { BES-A } \\
\text { Afetiva }\end{array}$ & $\begin{array}{c}\text { BES-A } \\
\text { Cognitiva }\end{array}$ \\
\hline \multicolumn{7}{|l|}{ Masculina } \\
\hline BES & 1 & & & & & \\
\hline BES Afetiva & $.87 * * *$ & 1 & & & & \\
\hline BES Cognitiva & $.79 * * *$ & $.39 * * *$ & 1 & & & \\
\hline BES-A & $.94 * * *$ & $.74 * * *$ & $.83 * * *$ & 1 & & \\
\hline BES-A Afetiva & $.76 * * *$ & $.89 * * *$ & $.32 * * *$ & $.76 * * *$ & 1 & \\
\hline BES-A Cognitiva & $.75 * * *$ & $.36 * * *$ & $.96 * * *$ & $.84 * * *$ & $.29 * * *$ & 1 \\
\hline \multicolumn{7}{|l|}{ Feminina } \\
\hline BES & 1 & & & & & \\
\hline BES Afetiva & $.86 * * *$ & 1 & & & & \\
\hline BES Cognitiva & $.79 * * *$ & $.37 * * *$ & 1 & & & \\
\hline BES-A & $.91 * * *$ & $.72 * * *$ & $.81 * * *$ & 1 & & \\
\hline BES-A Afetiva & $.71 * * *$ & $.84 * * *$ & $.26 * * *$ & $.74 * * *$ & 1 & \\
\hline BES-A Cognitiva & $.75 * * *$ & $.35 * * *$ & $.95 * * *$ & $.84 * * *$ & $.27 * * *$ & 1 \\
\hline
\end{tabular}

Quadro 5. Consistência interna da BES-A

\begin{tabular}{lcccc}
\hline Masculina/Feminina & Alfa & Omega & MCII & ACITC \\
\hline BES-A total & $.79 / .77$ & $.88 / .86$ & $.36 / .33$ & $.39-.61 / .37-.61$ \\
BES-A Afetiva & $.79 / .75$ & $.80 / .78$ & $.55 / .49$ & $.59-.72 / .45-.63$ \\
BES-A Cognitiva & $.84 / .84$ & $.85 / .84$ & $.57 / .57$ & $.60-.75 / .61-.72$
\end{tabular}

Nota. Alfa=Alfa de Cronbach; Omega=Coeficiente Omega; MCII=Média das correlações inter-item; ACITC=Amplitude das correlações item-total corrigidas

Quadro 6. Validade discriminante e associações com ansiedade social

\begin{tabular}{lccc}
\hline Masculina/Feminina & BES-A total & BES-A Afetiva & BES-A Cognitiva \\
\hline APSD & $-.02 /-.14 * *$ & $.06(.08) /-.03(.02)$ & $-.08(-.11 *) /-.17 * *(-.20 * * *)$ \\
YPI-S & $.04 /-.03$ & $.09 /(.10) /-.06(-.05)$ & $-.01(-.05) /-.01(-.01)$ \\
ICU & $-.32 * * * /-.35 * * *$ & $-.15 * *(-.09) /-.25 * * *(-.20 * * *)$ & $-.34 * * *(-.29 * * *) /-.30 * * *(-.25 * * *)$ \\
RPQ & $.10 /-.03$ & $.09(.09) /-.02(-.01)$ & $.05(.01) /-.04(-.03)$ \\
SAS-A & $.22 * * * / .06$ & $.34 * * *(.34 * * *) / .09(.11 *)$ & $.06(-.06) / .01(-.02)$ \\
\hline
\end{tabular}

Nota. BES-A=Escala de Empatia Básica versão breve; APSD=Dispositivo de Despiste de Processo Antissocial; YPIS=Inventário de Traços Psicopáticos para Jovens - versão breve; ICU=Inventário de Traços Calosos/Insensibilidade emocional; RPQ=Questionário de Agressividade Reativa-Proativa; SAS-A=Escala de Ansiedade Social para Adolescentes Correlações parciais controlando a outra dimensão da BES-A dadas em parêntesis $* * * p \leq .001 ; * * p \leq .01 ; * p \leq .05$

\section{Discussão}

O objetivo do presente estudo consistiu na adaptação de uma versão breve da BES, designada BES-A, em adolescentes portugueses. Os resultados revelaram que tanto a estrutura fatorial constituída por dois fatores de primeira ordem como a estrutura fatorial constituída por um fator de segunda ordem obtiveram bons índices de ajustamento nas diferentes amostras (Maroco, 2014; West et al., 2012). A comprovação de que a estrutura fatorial constituída por um fator de segunda ordem obtém bom ajustamento é especialmente interessante porque justifica a utilização uma pontuação total da BES-A, algo qua ainda não tinha sido evidenciado em estudos prévios feitos em Portugal (e.g., Anastásio et al., 2016; Pechorro et al., 2015, 2017).

Foi demonstrada invariância de medida fraca e forte entre rapazes e raparigas, o que permite a realização de comparações entre estes dois grupos (Chen, 2007). As correlações entre a BES-A total e as suas dimensões com a BES original e suas dimensões quer para o grupo masculino quer para o grupo feminino, apresentaram associações positivas que variaram de moderadas a altas (Ferguson, 2009), de acordo com o que era esperado quando se compara uma escala original com a sua versão breve. A nossa primeira hipótese foi portanto confirmada.

A consistência interna (fiabilidade) da BES-A, medida por alfa de Cronbach e coeficiente Omega, revelou valores de aceitáveis a bons 
sempre acima de 70 (Dunn et al., 2014; Nunnally, \& Bernstein, 1994) para a escala total e para as duas dimensões, o que é consistente com valores reportados em estudos prévios (e.g., Pechorro, Ray, Salas-Wright et al., 2015; Salas-Wright et al., 2012). A utilização do coeficiente Omega é recomendada dado que tem sido demonstrado que o alfa de Cronbach tende a subestimar a consistência interna (Dunn et al., 2014); apesar disso o alfa também foi fornecido dado que continua a ser o coeficiente tradicionalmente mais utilizado. As médias das correlações inter-itens revelaram valores adequados para a escala total (i.e., amplitude de .15 a .50), mas ligeiramente superiores ao desejável nas dimensões. As correlações item-total corrigidas (Clark \& Watson, 1995) revelaram valores adequados (acima de .30). A segunda hipótese foi portanto também confirmada.

A validade discriminante (Kaplan, \& Saccuzzo, 2013; Urbina, 2014) da BES-A com o APSD, o YPI-S, o ICU e o RPQ revelou de uma forma geral correlações negativas ou nãosignificativas conforme o esperado, em linha com investigações prévias (e.g., Dadds et al., 2009; Martorell, González, Ordóñez, \& Gómez, 2011; Muñoz, Qualter, \& Padgett, 2011; Vachon, Lynam, \& Johnson, 2014). Já as correlações com a SAS-A, mais especificamente da dimensão afetiva da BES-A, revelaram ser positivas baixas a moderadas e estatisticamente significativas. Tais correlações entre empatia afetiva e ansiedade social já haviam sido demonstradas noutros estudos, evidenciando que sujeitos com níveis altos de empatia afetiva tendem a ter níveis altos de ansiedade social (ver Tibi-Elhanany \& Shamay-Tsoory, 2011). Consideramos pois que a terceira hipótese foi confirmada.

Finalmente, em termos de validade de grupos conhecidos, as raparigas obtiveram sempre pontuações mais altas e estatisticamente significativas na BES-A total e suas dimensões, em consonância com a generalidade dos estudos feitos anteriormente (e.g., Eisenberg et al., 1999; Michalska, Kinzler, \& Decety, 2013). Confirmase desta forma a nossa hipótese final.

Devemos, todavia, mencionar algumas limitações da nossa investigação. Teria sido aconselhável a utilização de outras medidas validadas de empatia em adolescentes portugueses para analisar a validade convergente. Estudos futuros devem ser realizados com vista à obtenção de mais dados de natureza psicométrica sobre o instrumento (e.g., fiabilidade teste-reteste). Deverse-á igualmente proceder à validação cruzada noutras amostras (e.g., amostras clínicas) de forma a ter confiança de que as propriedades psicométricas se mantêm adequadas.

Concluímos que a versão breve da BES demonstrou propriedades psicométricas adequadas que justificam a sua utilização com adolescentes portugueses, nomeadamente em contexto escolar. Os resultados obtidos indicam que a medida breve aqui proposta constitui um instrumento de autorrelato válido e fiável para a avaliação da empatia em adolescentes. Os benefícios derivados da utilização da versão breve da BES são consideráveis a nível investigativo e de prática clínica dado que as versões breves das escalas têm diversas vantagens, entre as quais: demoram menos tempo a responder logo são menos fatigantes e menos aborrecidas para certas populações (e.g., adolescentes), diminuem a probabilidade de haver itens omissos, aumentam a validade facial na perspetiva dos participantes e são mais representativas do constructo em questão por manterem apenas os itens essenciais eliminado os repetidos ou muito semelhantes (Rammstedt \& Beierlein, 2014). Esperamos que este trabalho possa contribuir para fomentar novos trabalhos de investigação sobre empatia com adolescentes em diversos contextos.

\section{Referências}

Albiero, P., Matricardi, G., Speltri, D., \& Toso, D. (2009). The assessment of empathy in adolescence: A contribution to the Italian validation of the Basic Empathy Scale. Journal of Adolescence, 32, 393-408. doi:10.1016/j.adolescence.2008.01.001

Anastácio, S., Vagos, P., Nobre-Lima, L., Rijo, D., \& Jolliffe, D. (2016). The Portuguese version of the Basic Empathy Scale (BES): Dimensionality and measurement invariance in a community adolescent sample. European Journal of Developmental Psychology. 13, 614-623. doi:10.1080/17405629.2016.1167681

Ang, R. P., \& Goh, D. H. (2010). Cyberbullying among adolescents: The role of affective and 
cognitive empathy, and gender. Child Psychiatry \& Human Development, 41, 387397. doi:10.1007/s10578-010-0176-3

American Psychiatric Association. (2013). Diagnostic and statistical manual of mental disorders $\left(5^{\text {th }}\right.$ ed.). Washington, DC: Author.

Andershed, H., Kerr, M., Stattin, H., \& Levander, S. (2002). Psychopathic traits in non-referred youths: Initial test of a new assessment tool. In E. Blaauw, \& L. Sheridan (Eds.), Psychopaths: Current international perspectives (pp. 131-158). Haag, Netherlands: Elsevier.

Bentler, P., \& Wu, E. (2015). Supplement to EQS 6.3 for Windows user's guide. Temple City, CA: Multivariate Software, Inc.

Blair, R. J. R. (2005). Responding to the emotions of others: Dissociating forms of empathy through the study of typical and psychiatric populations. Consciousness and Cognition, 14, 698-718. doi:10.1016/j.concog.2005.06.004

Byrne, B. (2006). Structural equation modeling with EQS: Basic concepts, applications, and programming. Mahwah, NJ: Lawrence Erlbaum Associates.

Bryant, F., \& Satorra, A. (2012). Principles and practice of scaled difference chi-square testing. Structural Equation Modeling, 19, 372-398. doi:10.1080/10705511.2012.687671

Caputo, A., Frick, P., \& Brodsky, S. (1999). Family violence and juvenile sex offending. Criminal Justice and Behavior, 26, 338-356. doi: $10.1177 / 0093854899026003004$

Chaplin, T. M., \& Aldao, A. (2013). Gender differences in emotion expression in children: A meta-analytic review. Psychological Bulletin, 139, 735-765. doi:10.1037/a0030737

Chen, F. F. (2007). Sensitivity of goodness of fit indexes to lack of measurement invariance. Structural Equation Modeling, 14, 464-504. doi:10.1080/10705510701301834

Cheung, G. W., \& Rensvold, R. B. (2002). Evaluating goodness-of-fit indexes for testing measurement invariance. Structural Equation Modelling, $\quad 9, \quad 233-255$. doi:10.1207/S15328007SEM0902_5

Clark, L., \& Watson, D. (1995). Constructing validity: Basic issues in objective scale development. Psychological Assessment, 7, 309-319. doi:10.1037/1040-3590.7.3.309
Crocetti, E., Van der Graaff, J., Moscatelli, S., Keijsers, L., Koot, H., Rubini, M., ... \& Branje, S. (2016). A longitudinal study on the effects of parental monitoring on adolescent antisocial behaviors: The moderating role of adolescent empathy. Frontiers Psychology, 7, 1726. doi:10.3389/fpsyg.2016.01726

Dadds, M. R., Hawes, D. J., Frost, A. D., Vassallo, S., Bunn, P., Hunter, K., \& Merz, S. (2009). Learning to 'talk the talk': The relationship of psychopathic traits to deficits in empathy across childhood. Journal of Child Psychology and Psychiatry, 50, 599-606. doi:10.1111/j.14697610.2008.02058.x

D'Ambrosio, F., Olivier, M., Didon, D., \& Besche, C. (2009). The Basic Empathy Scale: A French validation of a measure of empathy in youth. Personality and Individual Differences, $\quad$ 46, 160-165. doi:10.1016/j.paid.2008.09.020

Davis, M. H. (1980). A multidimensional approach to individual differences in empathy. JSAS Catalog of Selected Documents in Psychology, 10, 1-85 (Retrieved from http://www.uv.es/ friasnav/Davis_1980.pdf).

Davis, M. H. (1983). Measuring individual differences in empathy: Evidence for a multidimensionalapproach. Journal of Personality and Social Psychology, 44, 113126. doi:10.1037/0022-3514.44.1.113

Decety, J. (2010). The neurodevelopment of empathy in humans. Developmental Neuroscience, $\quad 32, \quad 257-267$. doi:10.1159/000317771

Decety, J., \& Cowell, J. M. (2014). Friends or foes: Is empathy necessary for moral behavior? Perspectives on Psychological Science, $\quad 9, \quad 525-537$. doi:10.1177/1745691614545130

DeVellis, R.F. (1991). Scale development: Theory and applications. Newbury Park: Sage Publications.

Dunn, T., Baguley, T., \& Brunsden, V. (2014). From alpha to omega: A practical solution to the pervasive problem of internal consistency estimation. British Journal of Psychology, 105, 399-412. doi:10.1111/bjop.12046

Eisenberg, N., Guthrie, I. K., Murphy, B. C., Shepard, S. A., Cumberland, A., \& Carlo, G. (1999). Consistency and development of prosocial dispositions: A longitudinal study. 
Child Development, 70, 1360-1372. doi:10.1111/1467-8624.00100

Essau, C. A., Sasagawa, S., \& Frick, P. J. (2006). Callous-unemotional traits in a community sample of adolescents. Assessment, 13, 454469. doi:10.1177/1073191106287354

Ferguson, C. (2009). An effect size primer: A guide for clinicians and researchers. Professional Psychology: Research and Practice, 40, 532-538. doi:10.1037/a0015808

Forth, A., Kosson, D., \& Hare, R. (2003). Hare Psychopathy Checklist: Youth Version (PCL: $Y V)$ : Technical manual. Toronto: MultiHealth Systems.

Frick, P., \& Hare, R. (2001). The Antisocial Process Screening Device (APSD): Technical manual. Toronto, ON: Multi-Health Systems.

Geng, Y., Xia, D., \& Qin, B. (2012). The Basic Empathy Scale: A Chinese validation of a measure of empathy in adolescents. Child Psychiatry \& Human Development, 43, 499510. doi:10.1007/s10578-011-0278-6

Hambleton, R., Merenda, P., \& Spielberger, C. (2005). Adapting educational and psychological tests for cross-cultural assessment. Mahwah, NJ: Lawrence Erlbaum Associates.

Hare, R. (2003). Hare psychopathy checklistrevised $\left(2^{\text {nd }}\right.$ ed.). Toronto: Multi-Health Systems.

Heynen, E., van der Helm, G., Stams, G., \& Korebrits, A. (2016). Measuring empathy in a German youth prison: A validation of the German version of the Basic Empathy Scale (BES) in a sample of incarcerated juvenile offenders. Journal of Forensic Psychology Practice, $\quad 16, \quad 336-346$, doi:10.1080/15228932.2016.1219217

Hoffman, M. L. (1987). The contribution of empathy to justice and moral judgment. In N. Eisenberg, \& J. Strayer (Eds.), Empathy and its development (pp. 47-80). New York: Cambridge University Press.

Hogan, R. (1969). Development of an empathy scale. Journal of Consulting and Clinical Psychology, 33, 307-316. doi:10.1037/h0027580

IBM Corp. (2017). IBM SPSS Statistics for Windows (version 25). Armonk, NY: Author.

Jolliffe, D., \& Farrington, D. (2006). Development and validation of the Basic
Empathy Scale. Journal of Adolescence, 29, 589-611. doi:10.1016/j.adolescence.2005.08.010

Jolliffe, D., \& Farrington, D. (2011). Is low empathy related to bullying after controlling for individual and social background variables? Journal of Adolescence, 34, 59-71. Doi:10.1016/j.adolescence.2010.02.001

Kaplan, R., \& Saccuzzo, D. (2013). Psychological testing: Principles, applications, and issues ( $8^{\text {th }}$ ed.). Belmont, CA: Wadsworth, Cengage Learning.

Kimonis, E. R., Frick, P. J., Skeem, J. L., Marsee, M. A., Cruise, K., Munoz, L. C., ... \& Morris, A. S. (2008). Assessing callous-unemotional traits in adolescent offenders: Validation of the Inventory of Callous-Unemotional Traits. International Journal of Law and Psychiatry, 31, 241-252. doi:10.1016/j.ijlp.2008.04.002

La Greca, A., \& Lopez, N. (1998). Social anxiety among adolescents: Linkages with peer relations and friendships. Journal of Abnormal Child Psychology, 26, 83-94. doi:10.1023/A:1022684520514

Lam, C. B., Solmeyer, A. R., \& McHale, S. M. (2012). Sibling relationships and empathy across the transition to adolescence. Journal of Youth and Adolescence, 41, 1657-1670. doi:10.1007/s10964-012-9781-8

Leech, N., Barrett, K., \& Morgan, G. (2015). IBM SPSS for intermediate statistics: Use and interpretation $\left(5^{\text {th }}\right.$ ed.). New York: LEA.

Lipsey, M. W. (1998). Design sensitivity: Statistical power for applied experimental research. In L. Bickman \& D. J. Rog (Eds.), Handbook of applied social research methods (pp. 39-68). Thousand Oaks, CA: Sage.

Maroco, J. (2014). Análise de equações estruturais: Fundamentos teóricos, software \& aplicações. Pero Pinheiro: ReportNumber, Ltd.

Martorell, C., González, R., Ordóñez, A., \& Gómez, O. (2011). Estudio confirmatorio del cuestionario de conducta antisocial (CCA) y su relación con variables de personalidad y conducta antisocial. Revista Iberoamericana de Diagnóstico y Evaluación - e Avaliação Psicológica, 31(1), 97-114.

McClure, E. B. (2000). A meta-analytic review of sex differences in facial expression processing and their development in infants, children, and 
adolescents. Psychological Bulletin, 126, 424453. doi:10.1037/0033-2909.126.3.424

Mehrabian, A. (1996). Manual for the Balanced Emotional Empathy Scale (BEES). (Available from Albert Mehrabian, 1130 Alta Mesa Road, Monterey, CA, USA 93940).

Millsap, R., \& Olivera-Aguilar, M. (2012). Investigating measurement invariance using confirmatory factor analysis. In R. Hoyle (Ed.), Handbook of structural equation modeling (pp. 380-392). New York: The Guilford Press.

Michalska, K. J., Kinzler, K. D., \& Decety, J. (2013). Age-related sex differences in explicit measures of empathy do not predict brain responses across childhood and adolescence. Developmental Cognitive Neuroscience, 3, 22-32. doi:10.1016/j.dcn.2012.08.001

Muñoz, L., Qualter, P., \& Padgett, G. (2011). Empathy and bullying: Exploring the influence of callous-unemotional traits. Child Psychiatry \& Human Development, 42, 183196. doi:10.1007/s10578-010-0206-1

Nunnally, J., \& Bernstein, I. (1994). Psychometric theory $\left(3^{\text {rd }}\right.$ ed.). New York, NY: McGraw-Hill.

Pechorro, P., Andershed, H., Ray, J., Maroco, J., \& Gonçalves, R. (2015). Validation of the Youth Psychopathic Traits Inventory and Youth Psychopathic Traits Inventory - Short among incarcerated juvenile delinquents. Journal of Psychopathology and Behavioral Assessment, $\quad 37, \quad 576-586$. doi:10.1007/s10862-015-9490-1

Pechorro, P., Gonçalves, R. Hawes, S., \& Ray, J. (no prelo). Psychometric properties of two short versions of the Inventory of CallousUnemotional Traits among incarcerated youth. Journal of Emotional and Behavioral Disorders. doi:10.1177/1063426617717940

Pechorro, P., Ray, J., Gonçalves, R., \& Jesus, S. (2017). The Inventory of CallousUnemotional Traits: Psychometric properties among referred and non-referred Portuguese female juveniles. International Journal of Law and Psychiatry, 54, 67-75. doi:10.1016/j.ijlp.2017.05.002

Pechorro, P., Ayala-Nunes, L., Nunes, C., Maroco, J., \& Gonçalves, R. (2016). The Social Anxiety Scale for Adolescents: Measurement invariance and psychometric properties among a school sample of Portuguese youths. Child Psychiatry and Human Development, 47, 975-984. doi:10.1007/s10578-016-0627-6

Pechorro, P., Hidalgo, V., Nunes, C., \& Jiménez, L. (2016). Confirmatory factor analysis of the Antisocial Process Screening Device: SelfReport among incarcerated male juvenile offenders. International Journal of Offender Therapy and Comparative Criminology, 60, 1856-1872. doi:10.1177/0306624X15588903

Pechorro, P., Barroso, R., Maroco, J., Vieira, R., \& Gonçalves, R. (2015). Psychometric properties of the Psychopathy Checklist: Youth Version among Portuguese juvenile delinquents. International Journal of Offender Therapy and Comparative Criminology, 59, 1322-1337. doi:10.1177/0306624X14535558

Pechorro, P., Ray, J. V., Salas-Wright, C., Maroco, J., \& Gonçalves, R. A. (2015) Adaptation of the Basic Empathy Scale among a Portuguese sample of incarcerated juvenile offenders. Psychology, Crime and Law, 21, 699-714. doi:10.1080/1068316X.2015.1028546

Pechorro, P., Ribeiro da Silva, D., Rijo, D., Gonçalves, R. A., \& Andershed, H. (2017). Psychometric properties and measurement invariance of the Youth Psychopathic Traits Inventory - Short among Portuguese youths. Journal of Psychopathology and Behavioral Assessment, 39, 486-497. doi:10.1007/s10862-017-9597-7

Pechorro, P., Ray, J., Raine, A., Maroco, J., \& Gonçalves, R. (2017). The Reactive-Proactive Aggression Questionnaire: Validation among a Portuguese sample of incarcerated juvenile delinquents. Journal of Interpersonal Violence, $\quad 32, \quad$ 1995-2017. doi:10.1177/0886260515590784

Pechorro, P., Kahn, R., Ray, J., Raine, A., \& Gonçalves, R. A. (2017). Psychometric properties of the Reactive-Proactive Aggression Questionnaire among detained female juvenile delinquents and community youths. Criminal Justice and Behavior, 44, 531-550. doi:10.1177/0093854816686395

Pechorro, P., Gonçalves, R., Andershed, H., \& DeLisi, M. (no prelo). Female psychopathic traits in forensic and school context: 
Comparing the Antisocial Process Screening Device Self-Report and Youth Psychopathic Traits Inventory-Short. Journal of Psychopathology and Behavioral Assessment. doi:10.1007/s10862-017-9605-y

Pechorro, P., Kahn, R., Gonçalves, R., \& Ray, J. (2017). Psychometric properties of Basic Empathy Scale among female juvenile delinquents and school youths. International Journal of Law and Psychiatry, 55, 29-36. doi:10.1016/j.ijlp.2017.10.008

Pihet, S., Suter, M., Meylan, N., \& Schmid, M. (2014). Factor structure of the Youth Psychopathic Traits Inventory using the total score, three scale scores, and/or 10 subscale scores. Criminal Justice and Behavior, 41, 1214-1231. doi:10.1177/0093854814540287

Raine, A., Dodge, K., Loeber, R., Gatzke-Kopp, L., Lynam, D., Reynolds, C., ... \& Liu, J. (2006). The Reactive-Proactive Aggression Questionnaire: Differential correlates of reactive and proactive aggression in adolescent boys. Aggressive Behavior, 32, 159-171. doi:10.1002/ab.20115

Rammstedt, B., \& Beierlein, C. (2014). Can't we make it any shorter? The limits of personality assessment and ways to overcome them. Journal of Individual Differences, 35, 212220. doi:10.1027/1614-0001/a000141

Salas-Wright, C., Olate, R., \& Vaughn, M. (2012). Assessing empathy in Salvadoran high-risk and gang-involved adolescents and young adults: A Spanish validation of the Basic Empathy Scale. International Journal of Offender Therapy and Comparative Criminology, $\quad 57, \quad$ 1393-1416. doi:10.1177/0306624X12455170

Sánchez-Pérez, N., Fuentes, L., Jollife, D., \& González-Salinas, C. (2014). Assessing children's empathy through a Spanish adaptation of the Basic Empathy Scale: Parent's and child's report forms. Frontiers in Psychology, 5, 1-13 (article 1438). doi:10.3389/fpsyg.2014.01438

Singer, T., \& Lamm, C. (2009). The social neuroscience of empathy. The Year in Cognitive Neuroscience, 1156, 81-96. doi:10.1111/j.1749-6632.2009.04418.x

Skilling, T., Quinsey, V., \& Craig, W. (2001). Evidence of a taxon underlying serious antisocial behavior in boys. Criminal Justice and Behavior, 28, 450-470. doi:10.1177/009385480102800404

Tibi-Elhanany, Y., \& Shamay-Tsoory, S. (2011). Social cognition in social anxiety: First evidence for increased empathic abilities. Israel Journal of Psychiatry and Related Sciences, 48, 98-106.

Topçu, C., \& Erdur-Baker, Ö. (2012). Affective and cognitive empathy as mediators of gender differences in cyber and traditional bullying. School Psychology International, 33, 550561. doi:10.1177/0143034312446882

Urbina, S. (2014). Essentials of psychological testing $\left(2^{\text {nd }}\right.$ ed.). Hoboken, NJ: John Wiley \& Sons.

Vachon, D., Lynam, D., \& Johnson, J. (2014). The (non)relation between empathy and aggression: Surprising results from a metaanalysis. Psychological Bulletin, 140, 751773. doi:10.1037/a0035236

Van Baardewijk, Y., Andershed, H., Stegge, H., Nilsson, K., Scholte, E., \& Vermeiren, R. (2010). Development and tests of short versions of the Youth Psychopathic Traits Inventory and the Youth Psychopathic Traits Inventory-Child Version. European Journal of Psychological Assessment, 26, 122-128. doi:10.1027/1015-5759/a000017

Villadangos, M., Errasti, J., Amigo, I., Jolliffe, D., \& Garcia-Cueto, E. (2016). Characteristics of Empathy in young people measured by the Spanish validation of the Basic Empathy Scale. Psicothema, 28, 323-329. doi:10.7334/psicothema2016.6

West, S., Taylor, A., \& Wu, W. (2012). Model fit and model selection in structural equation modeling. In R. Hoyle (Ed.), Handbook of structural equation modeling (pp. 209-231). New York: The Guilford Press.

Zacarías-Salinas, X., \& Andrade-Palos, P. (2014). Una escala para evaluar prácticas parentales que promueven la conducta prosocial en preadolescentes. Revista Iberoamericana de Diagnóstico y Evaluación - e Avaliação Psicológica, 2, 117-135. 